\title{
Event-by-Event Study of Space-Time Dynamics in Flux-Tube Fragmentation
}

\author{
Cheuk-Yin Wong* \\ Physics Division, Oak Ridge National Laboratory, Oak Ridge, TN 37831, USA
}

\begin{abstract}
In the semi-classical description of the flux-tube fragmentation process for hadron production and hadronization in high-energy $e^{+} e^{-}$annihilations and $p p$ collisions, the rapidity-space-time ordering and the local conservation laws of charge, flavor, and momentum provide a set of powerful tools that may allow the reconstruction of the space-time dynamics of quarks and mesons in exclusive measurements of produced hadrons, on an event-by-event basis. We propose procedures to reconstruct the space-time dynamics from event-by-event exclusive hadron data to exhibit explicitly the ordered chain of hadrons produced in a flux tube fragmentation. As a supplementary tool, we infer the average space-time coordinates of the $q-\bar{q}$ pair production vertices from the $\pi^{-}$rapidity distribution data obtained by the NA61/SHINE Collaboration in $p p$ collisions at $\sqrt{s}=6.3$ to $17.3 \mathrm{GeV}$.
\end{abstract}

PACS numbers: $13.85 . \mathrm{Hd}$, 13.75.Cs, 13.66.Bc

\section{INTRODUCTION}

The fragmentation of a color flux tube is an important basic process in the production of low- $p_{T}$ particles in high-energy $e^{+} e^{-}$annihilations and $p p$ collisions [121. In the case of an $e^{+} e^{-}$annihilation, the initial reaction in $e^{+}+e^{-} \rightarrow q+\bar{q}$ leads to a color flux tube between the quark and the antiquark that subsequently fragments into produced hadrons. In a nucleon-nucleon collision, a quark of one nucleon and the diquark of the other nucleon form one flux tube, or its idealization as a quantum chromodynamics (QCD) string. Subsequent fragmentation of the flux tube leads to the production of low- $p_{T}$ hadrons. It is distinctly different from the process of relativistic hard-scattering and parton showering in perturbative QCD that dominate the production of the higher- $p_{T}$ hadrons 22 28].

The process of flux-tube fragmentation falls within the realm of non-perturbative QCD. A fundamental description of the process from the basic principles of QCD is still lacking. Simplified field theoretical descriptions in terms QED2 or QCD2 in one space and one time coordinates have been presented to understand some gross features of the process $[3,4,21,29,33$. However, phenomenological applications of the quantum field theoretical description remain quite limited.

The semi-classical description of the fragmentation process, on the other hand, has been quite successful phenomenologically [5 13]. Following Fig. 10 of [5], we show in Fig. 1 an example of the fragmentation of a $u-(u d)$ flux tube with an invariant mass of $\sqrt{s}=8.65 \mathrm{GeV}$ as implemented in the Lund model in PYTHIA 6.4 [11. One envisages in Fig. 1(b) a leading quark $u$ pulling apart from a leading diquark $u d$ at high energies. The vacuum is so polarized that ordered $\bar{q}_{i}-q_{i}$ pairs are produced at vertices $C_{i}$ inside the tube via the Schwinger pair-production mechanism [13. The interaction of a produced quark $q_{i}$ with an antiquark $\bar{q}_{i+1}$ produced in the adjacent vertex

\footnotetext{
*wongc@ornl.gov
}

leads to the production of a hadron (most likely a meson) as a yo-yo $h\left(q_{i} \bar{q}_{i+1}\right)$ state and the fragmentation of the flux tube. The rapidities of the produced chain of hadrons are ordered along the spatial longitudinal $z$-axis, and in time.

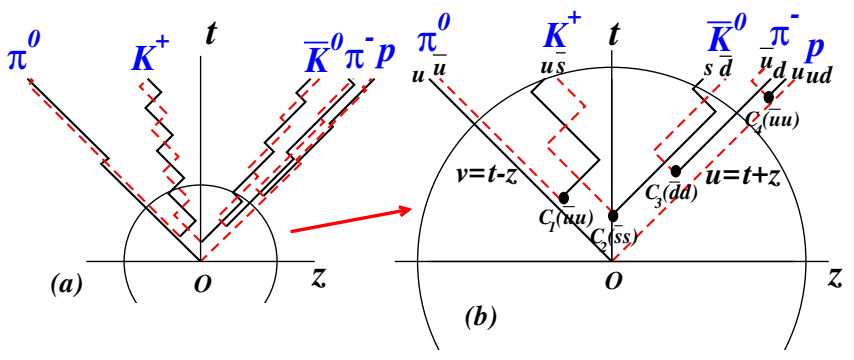

FIG. 1. An example of the space-time diagram in the fragmentation of a $u$ - $(u d)$ flux tube with an invariant mass of $\sqrt{s}=8.65 \mathrm{GeV}$, obtained with PYTHIA 6.4 [1]. Quark trajectories are shown as solid lines, and antiquark (or diquark) trajectories as dashed lines. Fig.1(b) is an expanded view of Fig.1(a). As the leading $u$ quark moves in the negative $z$-direction and the leading diquark $u d$ in the positive $z$ direction with nearly the speed of light, (massless) $\bar{q}_{i}-q_{i}$ pairs will be produced at vertices $C_{i}\left(u_{i}, v_{i}\right)$ lying approximately on a curve of $v=v(u)$, where $u=t+z$ and $v=t-z$. Subsequently, an antiquark $\bar{q}_{i}$ produced at one vertex $C_{i}$ interacts with the quark $q_{i+1}$ produced in the adjacent vertex $C_{i+1}$ to form a yo-yo $h\left(\bar{q}_{i} q_{i+1}\right)$ state which can be considered to represent a produced hadron.

While the semi-classical description of the flux-tube fragmentation process has been quite successful phenomenologically [5-13], a greater insight and a deeper understanding may be gained if the accompanying manybody hadronization dynamics can be tested on an eventby-event basis. In this respect, it is interesting to note that the semi-classical description provides a set of powerful tools to predict explicitly the many-body dynamics of the production process. Specifically, the process of flux-tube fragmentation is characterized by (i) a rapidityspace-time ordering of produced hadrons, (ii) the conservation of charge, flavor, and momentum for all $q-\bar{q}$ pair 
productions, and thus (iii) a complete correlation of all produced hadrons (mostly mesons and predominantly pions) in an orderly manner. If the particle production and hadronization process indeed follows such a semiclassical description, the above properties may allow the reconstruction of the configuration of the pair production space-time vertices of the flux tube. A chain of produced hadrons at the instances of fragmentation may be exhibited on an event-by-event basis, in exclusive (or nearly exclusive) measurements in which the momenta of almost all hadrons have been measured. Following Ref. [17, we suggest the use of event-by-event exclusive measurements to investigate the space-time many-body dynamics in flux-tube fragmentation in high energy $e^{+} e^{-}$ annihilations, $p p, p A$, and light $A A$ collisions.

Besides testing the basic contents of the semi-classical description of the many-body correlations, the measurements as proposed here will serve many other useful purposes. It is worth noting that direct experimental evidences for the number of flux tubes and the nature of the flux tubes in a $p p$ collision are still lacking. In the conventional Lund Model [11, a nucleon-nucleon collision is generally considered to consist of two colorless flux tubes. However, there are other models of particle production in terms of a more general color-singlet and non-colorsinglet flux tubes in a $p p$ collision, with different flux tube fragmentation properties. With an increase in the typical momentum transfer (or resolution power) in nucleonnucleon (and nucleus-nucleus) collisions at very high energies, the probability of finding gluons in a hadron (or a nucleus) increases, and the hadron can be described not only by the valence quarks and diquarks as in the Lund Model but also by a color object of predominantly gluons in the form of a color-glass condensate [35 40]. The fields in the region between the colliding gluon partons in a high energy $p p$ collision can be described by color flux tubes in the form of a glassma consisting of a region of non-zero color electric and color magnetic fields [35 40]. The decay of the glassma flux tubes may arise not from pair production but from the evolution of the classical Yang-Mills fields until the density is so low as to reach the hadronization limit 37, 38. The number of flux tubes in such a decay will depend on the gluon transverse density of the colliding hadrons and is yet to be empirically determined. The property of the fragmentation process of these tubes will need to be further investigated both theoretically and experimentally.

The explicit reconstruction of space-time dynamics of the many-body correlations proposed here may allow the identification and the enumeration of the fragmenting flux tubes to discrimnate between different models. If these flux tubes can be successfully identified in $p p$ collisions, they will provide additional experimental information on the nature of the flux tube, and the laws that govern the partition, the interference, and the interaction of the flux tubes. Furthermore, in more complicated $p A$ and $A A$ collisions involving a proton and light nuclei, the number of participating nucleons increases, and the dynamics of the flux tube fragmentation will display the effects of multiple collisions, the effects of the interaction between flux tubes, and the possibility of the merging of the flux tubes into ropes [20. It will stimulate future research on the quantum field description of the particle production process.

It is important to note that successes in the eventby-event studies carried out for flux-tube fragmentation will pave the way for future event-by-event investigations of the dynamics of other reaction mechanisms such as the hard-scattering process 22 28], direct fragmentation process 34, color-glass condensate 35-40, EPOS model [41, and Landau hydrodynamics 42 47. Each of these mechanisms exhibits specific space-time dynamics on an event-by-event basis and are important in different collision energies and $p_{T}$ domains. For example, if the collision dynamics in a $p p$ collision follow the space-time description of Landau hydrodynamics, the exact solution of Landau hydrodynamics in one space and one time directions 42, 43, 45] will predict the occurrence of a single tube of particles ordered in rapidity, instead of the two flux tubes in the flux-tube fragmentation model. If the hydrodynamic bulk matter freezes out into hadrons statistically and incoherently, then there will be no azimuthal back-to-back correlations and no flavor correlations between two hadrons adjacent in rapidity. In another example, a hard scattering process of two partons in a $p p$ collision 22 28 will result in a dijet consisting of two azimuthally back-to-back cones of produced hadrons on an event-by-event basis. These two cones will appear as two clusters of particles in the $(\eta, \phi)$ plane, centered at $\Delta \phi \sim \pi$ and at rapidities (or pseudorapidities) that depend on the initial longitudinal momenta of the colliding partons. The event-by-event dynamics of the produced particles can be utilized to study dijet and multiple dijet occurrences in $p p$ collisions. By identifying the particle production mechanism that has occurred in an event, one may discriminate different theoretical models and gain important pieces of information on the production and hadronization process. It is therefore useful to develop methods to analyze event-by-event dynamics of produced hadrons in order to extract the wealth of information they provide.

The importance of the color flux-tube fragmentation process relative to the hard-scattering process in $p p$ collisions depends on the collision energy and the $p_{T}$ domain [48. As the collision energy increases, the hardscattering process becomes relatively more and more dominant and the transverse momentum boundary $p_{T b}$ that separates the flux-tube fragmentation region from the hard-scattering region recedes to a smaller value of $p_{T}$ 48. There is some evidence of the dominance of flux-tube fragmentation for low- $p_{T}$ hadron production at central rapidity in $p p$ collisions at energies $\sqrt{s}=6$ to $200 \mathrm{GeV}$ from the study of two-hadron correlations data of the STAR and the NA61/SHINE Collaborations [4858. The $p p$ and $A A$ collisions in the NA61/SHINE and NICA energy range are particularly suitable for event- 
by-event studies of the space-time dynamics in flux-tube fragmentation.

It has recently been suggested that a knotted/linked network of flux tubes may be formed in the early cosmological evolution in a QCD-like phase transition that may provide the source for the inflation of the universe [59]. The knotty network of flux tubes explains why our universe has exactly three (large) spatial dimensions because flux tube knots are topologically stable only in exactly three space dimensions. In the late stages of the knotty inflation, the flux tube will fragment into smaller entities and the standard cosmological evolution begins. It is therefore of interest to study the space-time dynamics of the fragmentation of a flux tube to assist possible identification of flux-tube remnants in knotty inflation cosmological evolution.

This paper is organized as follows. In Section II, we discuss the event-by-event fluctuations and the underlying average features of the space-time dynamics that may be described semi-classically. Within the semi-classical description of flux-tube fragmentation, we derive the differential equation relating the forward and backward longitudinal light-cone coordinates $(u, v)$ of the $q-\bar{q}$ pairproduction vertices with the rapidity distribution $d N / d y$ of the observed hadrons. The equations can be further reduced into a set of coupled equations for $u$ and $v$ as a function of $y$. In Section III, we prove the property of the space-time-rapidity ordering for a general hadron rapidity distribution $d N / d y$. In Section IV, we solve for the space-time coordinates for many different common rapidity distributions. In Section V, we infer the spacetime coordinates from the rapidity distribution of $\pi^{-}$obtained by the NA61/SHINE Collaboration. We review the experimental signatures for flux-tube fragmentation in Section VI. In Section VII, we outline the procedures to carry out event-by-event exclusive measurements to study flux-tube fragmentation dynamics. In Section VIII and Appendix A, we discuss the experimental challenges in event-by-event studies and the heavier meson fractions as inferred from experimental data. In Section IX, we discuss the useful tools that may assist the reconstruction of the flux tubes in a $p p$ collision. In Section X, we study how the many-hadron correlations may distinguish the difference between the inside-outside or outside-inside cascades in the production of particles in flux-tube fragmentation. In Section XI, we present our conclusions and discussions.

\section{SPACE-TIME DYNAMICS IN FLUX-TUBE FRAGMENTATION}

It is clear from the outset that on an event-by event basis there will be high degrees of fluctuations and uncertainties in the dynamical variables of the produced hadrons in a flux-tube fragmentation. Nevertheless, each event will exhibit distinct dynamical characteristics and obey conservation laws that will presumably remain the same in different events. We can illustrate here some of these fluctuations, uncertainties, and conservation laws.

When basic quantum mechanics and the finite size are taken into account, the pair production mechanism manifests itself as a tunnelling phenomenon over a potential barrier [14. For a static field, the probability of pair production in a flux-tube fragmentation begins to become substantial only when the source quark and antiquark are separated at a distance greater than $d$, as determined by the masses $2 m$ of the produced particle pair and the string tension $\kappa$ [7, 14,

$$
d \geq \frac{2 m}{\kappa}
$$

There is thus an uncertainty of $\Delta z=d=2 \mathrm{~m} / \kappa$ in the spatial separation of the pair-production vertices due to quantum mechanics, in contrast to the semi-classical model with massless quarks where the pair of particles are produced at a single point. Furthermore, the probability of pair production exhibits oscillations as a function of the spatial locations, of the order of $10 \%$ percent in the central region, but substantially larger at the edges (Fig. 2 and 3 of [14]). When the transverse momentum of the produced fermion is included, the transverse momentum contributes to the transverse mass $m_{T}$ which replaces the mass $m$ in Eq. (1). Thus, the minimum production separation $d$ depends on the transverse momentum of the produced pair, and there will be additional fluctuations arising from the transverse momentum degree of freedom. In a similar manner, there will also be fluctuations associated with the flavor and strangeness degrees of freedom, as well as with the time-dependence of the pair-producing field.

In spite of these fluctuations, variations, and uncertainties, a flux-tube fragmentation will possess the characteristic pattern of a chain of hadrons, linking together as products of the flux tube. They are ordered in rapidity and correlated with their neighbors through the conservation of momentum, charge, and flavor in the pair production process. There will be an average space-time distribution around which the space-time coordinates of the produced $q-\bar{q}$ pair vertices will fluctuate. This average distribution can be identified as what can be obtained in the semi-classical description. For this purpose, we seek a relation between the space-time coordinates of the pairproduction vertices and the rapidity distribution of the produced hadrons in the semi-classical model.

In the quantum field theory of QED2, Casher et al. 3] showed that when a charged particle pulls away from its antiparticle with nearly the speed of light in the centerof-mass system, the dipole density of produced charged pairs is a Lorentz-invariant function and the lines of constant produced dipole density of charged pairs are hyperbolas with a constant proper time $\tau$. In a semi-classical picture of the fragmentation of a color flux tube, it can be shown that if all pair-production vertices of a fragmenting string fall on the curve of a constant proper production time $\tau_{\text {pro }}$, the rapidity distribution $d N / d y$ of the 
produced hadrons will be a constant given by [17, 21]

$$
\frac{d N}{d y}=\frac{\kappa \tau_{\text {pro }}}{m_{T}},
$$

where $m_{T}=\sqrt{m^{2}+p_{T}^{2}}$ is the transverse mass of a produced hadron (mostly likely a pion) with a rest mass $m$. Thus the presence of a rapidity plateau is an indication of the occurrence of the $q-\bar{q}$ production vertices along a curve of constant proper time, $\tau=\tau_{\text {pro }}$, represented by $u v=\tau_{\text {pro }}^{2}$. Here, the forward and backward light-cone coordinates $(u, v)$ are related to the space-time coordinates $(t, z)$ by

$$
\begin{aligned}
& u=t+z, \\
& v=t-z .
\end{aligned}
$$

As $(u, v)$ and $(t, z)$ are related by a simple linear transformation, we shall call both $(u, v)$ and $(t, z)$ as the spacetime coordinates. We can show further that in this case with boost invariance, the produced particles are ordered in rapidity and space-time [21]. The rapidity-space-time ordering of produced particles means that in the centerof-mass system, particles with a greater magnitude of rapidity $|y|$ are produced at a greater magnitude of the longitudinal coordinate $|z|$ and at a later time $t$.

Experimentally, the observed shape of $d N / d y$ of produced hadrons in $p p$ collisions is closer to a Gaussian distribution rather than a flat plateau distribution [46, 47, 54. The $q-\bar{q}$ production vertices are not expected to lie on the curve of a constant proper time. How do the space-time coordinates of the $q-\bar{q}$ production vertices depends on the hadron rapidity distribution $d N / d y$ ? If the $q-\bar{q}$ production vertices do not lie on the curve of a constant proper time, would the property of rapidityspace-time ordering of hadrons be maintained? Is the rapidity-space-time ordering of hadrons a general result that does not require the occurrence of a plateau structure in the hadron rapidity distribution $d N / d y$ ?

To answer these questions, we use $u$ as the independent variable and represent the locus of space-time lightcone coordinates $(u, v)$ of the pair-production vertices $C_{i}\left(u_{i}, v_{i}\right)$ in Fig. 1 by $v(u)$ as a function of $u$. We would like to write down the differential equation governing $v(u)$ in terms of $d N / d y$.

The rapidity of a hadron yo-yo state $y_{i}$ is related to the space-time coordinates of its constituent quark and antiquark that have been produced at vertices $C_{i-1}=\left(u_{i-1}, v_{i-1}\right)$ and $C_{i}=\left(u_{i}, v_{i}\right)$ by

$$
y_{i}=\frac{1}{2} \ln \frac{\kappa\left(u_{i+1}-u_{i}\right)}{\kappa\left(v_{i}-v_{i+1}\right)} .
$$

Therefore, we have

$$
\frac{\Delta N}{\Delta y}=\frac{1}{y_{i}-y_{i-1}}=2 /\left[\ln \left(\frac{u_{i}-u_{i+1}}{v_{i+1}-v_{i}}\right)-\ln \left(\frac{u_{i-1}-u_{i}}{v_{i}-v_{i-1}}\right)\right] .
$$

Upon taking the continuum limits of the above two equations (4) and (5), we get 21]

$$
\begin{aligned}
y & =-\frac{1}{2} \ln \left(-\frac{d v}{d u}\right), \\
\frac{d N}{d y} & =\frac{2 \kappa}{m_{T}}\left(-\frac{d v}{d u}\right)^{3 / 2}\left(\frac{d^{2} v}{d u^{2}}\right)^{-1},
\end{aligned}
$$

where we have used the relation between $\Delta u_{i}=u_{i+1}-u_{i}$, $\Delta v_{i}=v_{i+1}-v_{i}$, and the transverse mass $m_{T i}$ of the produced hadron, $\Delta u_{i}\left(-\Delta v_{i}\right)=\left(\Delta u_{i}\right)^{2}(-d v / d u)=m_{T i}^{2} / \kappa^{2}$. From Eq. (7), the differential equation that governs $u(v)$ is related directly to the hadron rapidity $d N / d y$ by

$$
\frac{d^{2} v}{d u^{2}}=\frac{2 \kappa}{m_{T}(d N / d y)}\left(-\frac{d v}{d u}\right)^{3 / 2} .
$$

If $d N / d y$ is a known function of $y$, then Eqs. (6) and (8) can be used to solve for $v(u)$.

We can re-write Eqs. (6) and (8) in a simpler form to facilitate the solution of $u$ and $v$ for a general $d N / d y$. From Eq. (6), we have

$$
-\frac{d v}{d u}=e^{-2 y}
$$

Substituting this into Eq. (8), we obtain

$$
\frac{d y}{d u}=\frac{\kappa}{m_{T}(d N / d y)} e^{-y},
$$

which gives

$$
\frac{d u}{d y}=\frac{m_{T}}{\kappa} \frac{d N}{d y} e^{y} .
$$

Using $d v / d y=(d v / d u)(d u / d y)$ and Eq. (9) we obtain the equation of $d v / d y$

$$
\frac{d v}{d y}=-\frac{m_{T}}{\kappa} \frac{d N}{d y} e^{-y} .
$$

The set of Eqs. (11) and (12) allow one to solve for the space-time coordinates $(u, v)$ and $(t, z)$ of the $q-\bar{q}$ pairproduction vertices as a function of the rapidity variable $y$, for a general hadron rapidity distribution $d N / d y$.

\section{RAPIDITY-SPACE-TIME ORDERING OF THE PRODUCED HADRONS}

We can infer some important gross properties of the rapidity-space-time ordering from Eqs. (11) and (12). We can evaluate $d y / d z$ and we get

$$
\begin{aligned}
\frac{d y}{d z} & =\frac{d y}{d u} \frac{d u}{d z}+\frac{d y}{d v} \frac{d v}{d z} \\
& =\frac{m_{T}}{\kappa} \frac{d N}{d y} 2 \cosh y .
\end{aligned}
$$

Because all factors on the right-hand side of the above equation are positive, we have

$$
\frac{d y}{d z}>0 \text {. }
$$


This says that the rapidities of the produced hadrons are ordered in longitudinal space. Those hadrons with a greater rapidity $y$ are produced at a greater value of the $z$ coordinate. We can similarly evaluate $d y / d t$,

$$
\begin{aligned}
\frac{d y}{d t} & =\frac{d y}{d u} \frac{d y}{d t}+\frac{d y}{d v} \frac{d v}{d t} \\
& =\frac{m_{T}}{\kappa} \frac{d N}{d y} 2 \sinh y,
\end{aligned}
$$

which says that

$$
\begin{cases}d y / d t<0 & \text { for } y<0 \\ d y / d t=0 & \text { for } y=0 \\ d y / d t>0 & \text { for } y>0\end{cases}
$$

Combining the above results on $d y / d z$ and $d y / d t$ in Eqs. (14) and (18), we obtain the rapidity-space-time ordering of produced hadrons. The hadrons with a greater magnitude of rapidity $|y|$ are produced at a greater magnitude of the longitudinal coordinate $|z|$ and at a later time $t$.

We have thus proved the result of the rapidity-spacetime ordering for a general rapidity distribution $d N / d y$ in a semi-classical description of flux-tube fragmentation, provided the vertices of the produced $q-\bar{q}$ pairs lie on a curve of $v=v(u)$. It should be pointed out that the production of a $q-\bar{q}$ pair is a stochastic process, whose occurrence has a probability distribution and fluctuations. The coordinates of the vertices should fluctuate about an average distribution on an event-by-event basis.

\section{SOLUTION FOR THE SPACE-TIME COORDINATES OF PAIR-PRODUCTION VERTICES FOR A GIVEN $d N / d y$}

Given a hadron rapidity distribution $d N / d y$ that is a function of $y$, Eqs. (11) and (12) can be integrated out to give a parametric representation of the space-time coordinates $(u, v)$ and $(z, t)$ of the $q-\bar{q}$ production vertices. They assume analytical forms for many common hadron rapidity distributions $d N / d y$, including a boost-invariant, a plateau distribution, and a Gaussian distribution.

\section{A. Space-time vertex coordinates for a boost-invariant $d N / d y$}

We consider first the case of a boost-invariant $d N / d y$ that is independent of the rapidity. The distribution can be represented by

$$
\frac{d N}{d y}=(d N / d y)_{0},
$$

where $(d N / d y)_{0}$ is $d N / d y$ at $y=0$. In terms of the average transverse mass $m_{T}$ and the string constant $\kappa$, we can introduce $\tau_{0}$ given as in Eq. (2) by

$$
\tau_{0}=\tau_{\text {pro }}=\frac{m_{T}}{\kappa}(d N / d y)_{0} .
$$

Eqs. (11) and 12 then yield the solution of the spacetime coordinates

$$
\begin{aligned}
& u(y)=\tau_{0} e^{y} \\
& v(y)=\tau_{0} e^{-y},
\end{aligned}
$$

and

$$
\begin{aligned}
& t=\tau_{0} \cosh y \\
& z=\tau_{0} \sinh y
\end{aligned}
$$

As a consequence,

$$
u(y) v(y)=\tau^{2}=t^{2}-z^{2}=\tau_{0}^{2} .
$$

This is the well-known result that the boost-invariant pair-production vertices lie, on the average, on a curve of constant proper time, $\tau=\tau_{0}=\tau_{\text {pro }}$. It occurs only for the case in the fragmentation of a flux tube with infinitely large energies.

\section{B. Space-time vertex coordinates for a plateau rapidity distribution $d N / d y$}

For the fragmentation of a flux tube with a finite energy, we consider next the more realistic case of a rapidity distribution with a plateau structure within a limited region of $|y| \leq y_{\max }$,

$$
\frac{d N}{d y}=(d N / d y)_{0} \Theta\left(y_{\max }-|y|\right) .
$$

We can again represent $\left(m_{T} / \kappa\right)(d N / d y)_{0}$ by $\tau_{0}$ as in Eq. (18). Equations (11) and (12) yield the solution for $|y| \geq y_{\max }$,

$$
\begin{aligned}
& u(y)=\tau_{0} e^{y} \\
& v(y)=\tau_{0} e^{-y},
\end{aligned}
$$

and

$$
\begin{aligned}
& t=\tau_{0} \cosh y \\
& z=\tau_{0} \sinh y .
\end{aligned}
$$

In this case with a rapidity plateau distribution, the ranges of $t$ and $z$ are limited, with $\tau_{0} \leq t \leq \tau_{0} \cosh y_{\max }$ and $|z| \leq \tau_{0} \sinh y_{\text {max }}$.

\section{Space-time vertex coordinates for a Gaussian $d N / d y$}

We consider the fragmentation of a flux tube which gives rise to the production of hadrons with the Gaussian rapidity distribution centering at the rapidity $y_{0}$,

$$
\frac{d N_{\mathrm{FT}}}{d y}=N_{\mathrm{FT}} \frac{\exp \left\{-\frac{\left(y-y_{0}\right)^{2}}{2 \sigma^{2}}\right\}}{\sqrt{2 \pi} \sigma},
$$


where $N_{\mathrm{FT}}$ is the integrated number of hadrons (predominantly pions) produced per flux tube. The equation for $d u / d y$, Eq. (11), becomes

$$
\frac{d u}{d y}=\frac{m_{T}}{\kappa} \frac{N_{\mathrm{FT}}}{\sqrt{2 \pi} \sigma} e^{-\frac{1}{2 \sigma^{2}}\left(y-y_{0}\right)^{2}} e^{y} .
$$

This differential equation can be solved to give the solution

$u(y)=e^{y_{0}}\left[\frac{m_{T}}{\kappa} \frac{N_{\mathrm{FT}} e^{\sigma^{2} / 2}}{2}\left\{1+\operatorname{erf}\left(\frac{y-y_{0}-\sigma^{2}}{\sqrt{2} \sigma}\right)\right\}\right]$

The equation for $d v / d y$, Eq. 12, is

$$
\frac{d v}{d y}=-\frac{m_{T}}{\kappa} \frac{N_{\mathrm{FT}}}{\sqrt{2 \pi} \sigma} e^{-\frac{1}{2 \sigma^{2}}\left(y-y_{0}\right)^{2}} e^{-y},
$$

which can also be solved to give the solution

$$
v(y)=e^{-y_{0}}\left[\frac{m_{T}}{\kappa} \frac{N_{\mathrm{FT}} e^{\sigma^{2} / 2}}{2}\left\{1-\operatorname{erf}\left(\frac{y-y_{0}+\sigma^{2}}{\sqrt{2} \sigma}\right)\right\}\right]
$$

We can form $t=(u+v) / 2$ and $z=(u-v) / 2$ to yield

$$
\begin{array}{r}
t=\frac{m_{T}}{\kappa} \frac{N_{\mathrm{FT}} e^{\sigma^{2} / 2}}{4}\left[e^{y_{0}}\left\{1+\operatorname{erf}\left(\frac{y-y_{0}-\sigma^{2}}{\sqrt{2} \sigma}\right)\right\}\right. \\
\left.+e^{-y_{0}}\left\{1-\operatorname{erf}\left(\frac{y-y_{0}+\sigma^{2}}{\sqrt{2} \sigma}\right)\right\}\right], \\
z=\frac{m_{T}}{\kappa} \frac{N_{\mathrm{FT}} e^{\sigma^{2} / 2}}{4}\left[e^{y_{0}}\left\{1+\operatorname{erf}\left(\frac{y-y_{0}-\sigma^{2}}{\sqrt{2} \sigma}\right)\right\}\right. \\
\left.-e^{-y_{0}}\left\{1-\operatorname{erf}\left(\frac{y-y_{0}+\sigma^{2}}{\sqrt{2} \sigma}\right)\right\}\right] .
\end{array}
$$

The above two equations give the parametric representation of the space-time coordinates $t$ and $z$ of the produced $q-\bar{q}$ pairs leading to the production of hadrons with the Gaussian rapidity distribution Eq. 25).

\section{SPACE-TIME COORDINATES OF $q-\bar{q}$ PRODUCTION VERTICES INFERRED FROM NA61/SHINE $d N_{p p}\left(\pi^{-}\right) / d y$ DATA}

The results in the last section indicates that the spacetime coordinate of $q-\bar{q}$ production vertices are directly related to the hadron rapidity distribution. We need the experimental hadron rapidity distribution data $d N / d y$ to infer the space-time coordinates of $q-\bar{q}$ production vertices in flux-tube fragmentation. As the experimental $d N / d y$ data represent the rapidity distribution averaged over many events, the space-time coordinates inferred from the experimental $d N / d y$ data should also be considered as space-time coordinates averaged over an ensemble of events.

In $p p$ collisions at energies investigated by the NA61/SHINE Collaboration, the flux-tube fragmentation process occurs in the central rapidity region, whereas target and projection fragmentation processes take place near the projectile and target rapidities, respectively. As a consequence, positive (positively charged) hadrons can be produced in all three regions of the rapidity space in $p p$ collisions. On the other hand, low- $p_{T}$ negative (negatively charged) pions are unlikely to be produced in the projectile and target fragmentation regions in $p p$ collisions, and can be appropriately considered to originate predominantly from the flux-tube fragmentation process in the central rapidity region without contributions from projectile and target fragmentations.

The NA61/SHINE Collaboration has measured the transverse and longitudinal spectra of negative pions in the collision of a proton on a fixed proton target at $p_{\text {lab }}=20,31,40,80$, and $158 \mathrm{GeV} / \mathrm{c}$ 54]. The transverse spectra of low- $p_{T}$ pions can be represented by $d N / m_{T} d m_{T} \propto \exp \left\{-m_{T} / T\right\}$ where $T$ ranges from 150 to $160 \mathrm{MeV}$ [54. After integrating over the transverse spectra, the NA61/SHINE $\pi^{-}$data of the rapidity distribution for different $p_{\text {lab }}$ are shown in Fig. 2

Our event-by-event study has been motivated to obtain a space-time description of the $p p$ collisions process for which direct experimental information remains lacking. Different models have been presented for such a description with varying degrees of successes, but they will need to be tested with direct event-by-event measurements which we envisage here.

The most common description for the flux tube fragmentation in a $p p$ collision is given in terms by the Lund Model [5 12]. It is assumed that in the relatively lower energy region in $p p$ collisions, the valence quarks and valence diquarks of the colliding protons dominates the soft particle production process. By color conservation, the only simpest colorless objects formed by the valence consituents of one nucleon and the valence consituents of the other nucleon after the collision are two colorless flux tubes formed by the valence quark of one proton and the valence diquark of the other colliding proton. Each colorless flux tube undergoes fragmention in the same way as in an $e^{+}-e^{-}$annihilation. At higher $p p$ (and $A A$ ) collision energies, there are other models of particle production in terms of a more general color-singlet and non-color-singlet flux tubes involving also gluons in additon to valence quark and valence diquarks, with different flux tube fragmentation properties. For example, at high-multiplicity events at very high energies, the possibility of forming more than two flux tubes has been suggested [10. In another example at very high energies, the probability of finding gluons in a hadron (or a nucleus) increases, and the hadron can be described by a color object of predominantly gluons in the form of a color-glass condensate in additon to valence quark and valence diquarks [35 40]. The fields in the region between the colliding gluon partons after a high energy $p p$ collision can be described by glassma flux tubes 35-40. whose number depends on the gluon transverse density of the colliding hadrons. The longitudinal expansion of the glassma flux tubes as the classical Yang-Mill field evolves 
to lower densities leads to the fragmentation of the tubes.

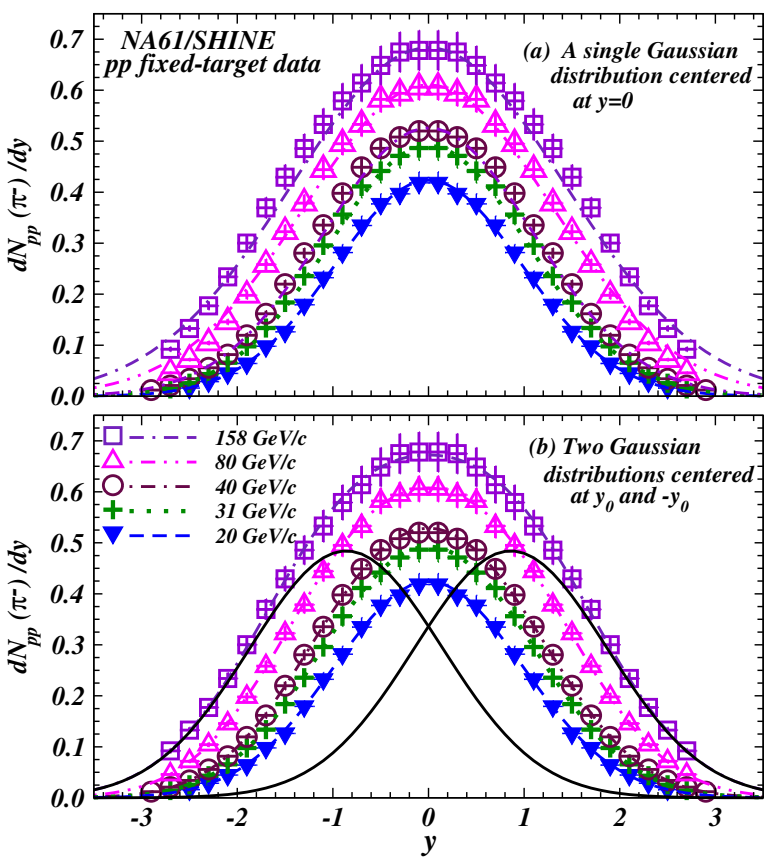

FIG. 2. (Color online). The data points are NA61/SHINE $d N_{p p}\left(\pi^{-}\right) / d y$ data for different $p_{\text {lab }}$ [54. The curves in Fig. (a) give the fits to the data points using a single Gaussian rapidity distribution, Eq. $(32)$, centering at $y=0$, with parameters in Table I. The curves in Fig. (b) give fits to the data points using the sum of two displaced Gaussian distributions, Eq. (34), centering at $+y_{0}$ and $-y_{0}$, with parameters in Table II. The two solid curves in Fig. (2b) give the two separate Gaussian distributions at $+y_{0}$ and $-y_{0}$ for the case of $p_{\text {lab }}=158 \mathrm{GeV}$.

As the number of flux tubes and their properties in a $p p$ collision are not yet known experimentally, we shall analyze the production of the low- $p_{T}$ negative pions by assuming two possible scenarios with the occurrence of (I) a single flux tube, or (II) two differently-displaced flux tubes. The scenarios of many more flux tubes can be similarly generalized. The proper description to distinguish between the different scenarios may need to rely on experimental information that will be forthcoming in the event-by-event analysis.

With the assumption of the scenario (I) of a single flux tube, we can represent the rapidity distribution by a single Gaussian distribution

$$
\frac{d N_{p p}\left(\pi^{-}\right)}{d y}=\frac{\left\langle\pi^{-}\right\rangle}{\sqrt{2 \pi} \sigma} \exp \left\{-\frac{y^{2}}{2 \sigma^{2}}\right\}
$$

The fits of the experimental NA61/SHINE $\pi^{-}$rapidity distribution data in terms of a single-Gaussian distribution are shown in Fig. 2(a). The fitting parameter $\left\langle\pi^{-}\right\rangle$ is the average number of $\pi^{-}$produced per $p p$ collision, related to the total number of pions produced per flux tube $N_{\text {FT }}$ by

$$
N_{F T} \sim 3\left\langle\pi^{-}\right\rangle / 2
$$

and $\sigma$ is the standard deviation of the distribution. They are listed as a function of the incident $p_{\text {lab }}$ in Table I.

TABLE I. Parameters of the single-Gaussian fit (Eq. (32) to the NA61/SHINE $\pi^{-}$rapidity data in $p p$ collisions [54].

\begin{tabular}{|c|c|c|c|}
\hline$p_{\text {lab }}(\mathrm{GeV} / \mathrm{c})$ & $\sqrt{s}(\mathrm{GeV})$ & $\left\langle\pi^{-}\right\rangle$ & $\sigma$ \\
\hline 20 & 6.25 & $1.04 \pm 0.05$ & $0.98 \pm 0.030$ \\
31 & 7.75 & $1.28 \pm 0.06$ & $1.04 \pm 0.035$ \\
40 & 8.77 & $1.45 \pm 0.05$ & $1.10 \pm 0.044$ \\
80 & 12.33 & $1.99 \pm 0.08$ & $1.30 \pm 0.052$ \\
158 & 17.27 & $2.45 \pm 0.13$ & $1.43 \pm 0.065$ \\
\hline
\end{tabular}

To go from the rapidity distribution to the average space-time coordinates of the pair-production vertices, we need a model to relate these quantities. For definiteness, we shall provide predictions on the space-time behavior based on the semi-classical description of the particle production in a flux tube fragmentation in the Lund Model [5-11] in Section IVC, so that experimental deviations from such a semi-classical description, if any, will provide impetus for a search for the other alternative descriptions and models. We assume a string tension $\kappa=1 \mathrm{GeV} / \mathrm{fm}$, and the average transverse mass $m_{T}=2 T$ from the slope parameters $T$ of the transverse spectra in 54 58. From Eqs. (25)-(31), we obtain the space-time coordinates of $q-\bar{q}$ production vertices for different $p_{\text {lab }}$ shown in Fig. 3(a), under the assumption of scenario (I) of a single flux tube in $p p$ collisions. As $p_{\text {lab }}$ increases, the curve of the space-time coordinates rises higher in $t$ and extends farther in $|z|$

Previously, with an additional parameter, the NA61/SHINE Collaboration partitions the $\pi^{-}$rapidity distribution as the sum of two symmetrically displaced Gaussian distributions centering at $y_{0}$ and $-y_{0}$ [54],

$$
\begin{aligned}
\frac{d N_{p p}\left(\pi^{-}\right)}{d y}=\frac{\left\langle\pi^{-}\right\rangle}{2} & \left\{\frac{1}{\sqrt{2 \pi} \sigma} \exp \left(-\frac{\left(y-y_{0}\right)^{2}}{2 \sigma^{2}}\right)\right. \\
& \left.+\frac{1}{\sqrt{2 \pi} \sigma} \exp \left(-\frac{\left(y+y_{0}\right)^{2}}{2 \sigma^{2}}\right)\right\} .
\end{aligned}
$$

The values of the average number of $\pi^{-}$produced per $p p$ collision $\left\langle\pi^{-}\right\rangle$, the rapidity displacement $y_{0}$, and the standard deviation $\sigma$ of the rapidity distribution are tabulated for different values of $p_{\text {lab }}$ in Table II (from Table 5 of Ref. 54). The fits to the rapidity distributions using two displaced Gaussian distributions in Eq. (34) are shown in Fig. 2(b). The rapidity distributions with two displaced Gaussian distributions give a better agreement than a single Gaussian at the tail regions.

Comparing Table I and II, one notices that $\left\langle\pi^{-}\right\rangle$are nearly the same in the two tables. For the case of two displaced Gaussian distributions, the displacement $y_{0}$ increases substantially as $p_{\text {lab }}$ increases, but the standard deviation $\sigma$ changes only slightly. The increase in $y_{0}$ for two displaced Gaussian distributions shows up as an increase in $\sigma$ for a single Gaussian distribution.

The success in the description with two displaced Gaussian rapidity distributions suggests the alternative 

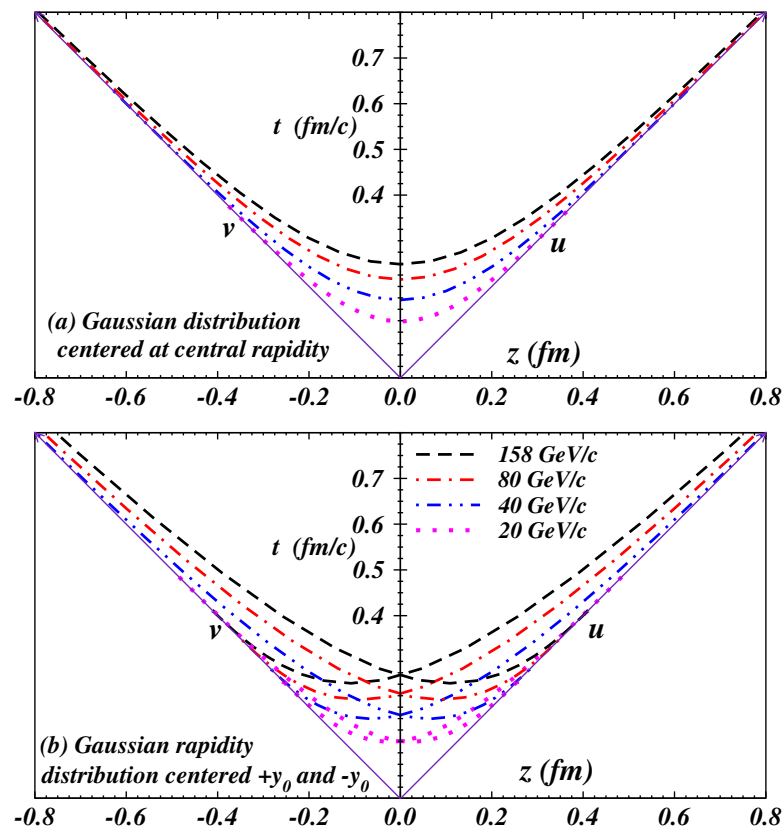

FIG. 3. (Color online). The locus of average space-time coordinates of $q-\bar{q}$ production vertices for different $p_{\text {lab }}$ as inferred from the NA61/SHINE $\pi^{-}$rapidity distribution data. (a) under the assumption (I) of the occurrence of a single flux tube and a Gaussian rapidity distribution, Eq. (32), centering at zero, and (b) under the assumption (II) of two flux tubes and two displaced Gaussian rapidity distributions, Eq. (34), centering at $+y_{0}$ and $-y_{0}$.

TABLE II. Parameters of the two-displaced-Gaussian fit (Eq. (34)) to the NA61/SHINE $\pi^{-}$rapidity data in $p p$ collisions, from Ref. 54.

\begin{tabular}{|c|c|c|c|}
\hline$p_{\text {lab }}(\mathrm{GeV} / \mathrm{c})$ & $\left\langle\pi^{-}\right\rangle$ & $\sigma$ & $y_{0}$ \\
\hline 20 & $1.047 \pm 0.051$ & $0.921 \pm 0.118$ & $0.337 \pm 0.406$ \\
31 & $1.312 \pm 0.069$ & $0.875 \pm 0.050$ & $0.545 \pm 0.055$ \\
40 & $1.478 \pm 0.051$ & $0.882 \pm 0.045$ & $0.604 \pm 0.044$ \\
80 & $1.938 \pm 0.080$ & $0.937 \pm 0.019$ & $0.733 \pm 0.010$ \\
158 & $2.444 \pm 0.130$ & $1.007 \pm 0.051$ & $0.860 \pm 0.021$ \\
\hline
\end{tabular}

scenario (II) of two flux tubes in a $p p$ collision, in which the fragmentation process takes place between the diquark of one nucleon and a valance quark of the other nucleon. The displaced Gaussian rapidity distribution arises from the asymmetry between the diquark and the valence quark in each flux tube. For this case, Eqs. (30) and (31) give the parametric representation of the average space-time coordinates $(t, z)$ of the vertices of $q-\bar{q}$ pair production in Fig. 3(b). As one observes, the spacetime coordinates of the pair-production vertices in one of the two flux tubes in a $p p$ collision is asymmetrical with respect to the $t$ axis, arising from the rapidity displacement $y_{0}$. As the collision momentum $p_{\text {lab }}$ increases, the magnitude of the rapidity displacement $y_{0}$ increases, and the asymmetry increases. The space-time coordinates of the pair-production vertices rises up as a function of $t$ and are shifted to greater values of $z$, There is another symmetrically displaced flux tube with hadron rapidity displacement at $-y_{0}$. The corresponding space-time coordinates of the pair-production vertices can be obtained by a reflection with respect to the $t$ axis.

It should be emphasized again that what we have shown in Fig. 3(a) or 3(b) represents the behavior of the space-time coordinates averaged over an ensemble of events. The production of a $q-\bar{q}$ pair is a stochastic process with fluctuations. The event-by-event locations of space-time coordinates of $q-\bar{q}$ production vertices are expected to fluctuate around the average space-time coordinates obtained in Fig. 3.

\section{EXPERIMENTAL TWO-HADRON CORRELATION SIGNATURE OF FLUX-TUBE FRAGMENTATION}

In a flux-tube fragmentation, the production of quarkantiquark pairs along a color flux tube precedes the fragmentation of the tube. Because of local conservation laws, the production of a $q-\bar{q}$ pair will lead to correlations of adjacently produced hadrons (mostly pions). On account of the rapidity-space-time ordering of produced mesons in a flux-tube fragmentation discussed in Section III, adjacently produced mesons can be signaled by their rapidity difference $\Delta y$ falling within the window of $|\Delta y|<1 /(d N / d y)$. Therefore, the local conservation laws of momentum, charge, and flavor will lead to a suppression of the angular correlation function $d N /(d \Delta \phi d \Delta y)$ for two hadrons with opposite charges or strangeness on the near side at $(\Delta \phi, \Delta y) \sim 0$, but an enhanced correlation on the back-to-back, away side at $\Delta \phi \sim \pi$, within the window of $|\Delta y|<1 /(d N / d y)$. These properties can be used as signatures for the fragmentation of a color flux tube [48.

For $p p$ collisions at $\sqrt{s_{p p}}=200 \mathrm{GeV}$, the STAR Collaboration found that if one separates the transverse momentum regions by the boundary $p_{T b}=0.5 \mathrm{GeV} / \mathrm{c}$, the two-hadron angular correlation pattern, $\Delta \rho / \sqrt{\rho_{\text {ref }}} \propto$ $d N / d \Delta \phi d \Delta \eta$, for two oppositely charged hadrons in the domain below $p_{T}<p_{T b}$ is distinctly different from the pattern in the domain above $p_{T}>p_{T b}$ [49 53. One finds a suppression at $(\Delta \eta, \Delta \phi) \sim 0$ but an enhancement at $(\Delta \eta \sim 0, \Delta \phi \sim \pi)$. Such a pattern is consistent with the theoretical correlation pattern for two oppositely charged hadrons for a flux-tube fragmentation [48. In the higher$p_{T}$ domain of $p_{T b}>0.5 \mathrm{GeV} / \mathrm{c}$, the correlation pattern is consistent with the hard-scattering process with the production of two jets (minijets) 48.

The NA61/SHINE Collaboration has reported the angular correlation for two hadrons with opposite charges and $p_{T}<1.5 \mathrm{GeV} / \mathrm{c}$, for $p p$ collisions at $\sqrt{s_{p p}}=6-17$ $\mathrm{GeV}$ [55 58. The experimental correlation patterns show a suppression at $(\Delta \phi, \Delta \eta) \sim 0$, and an enhancement at $(\Delta \eta \sim 0, \Delta \phi \sim \pi)$, indicating the dominance of the flux-tube fragmentation in the low- $p_{T}$ domain. Such a 
two-hadron correlation pattern remains unchanged up to $p_{T}=1.5 \mathrm{GeV} / \mathrm{c}$ [55]. The pattern of the NA61/SHINE two-hadron correlation was also shown to be qualitatively consistent with the EPOS model [41, 55]. Although there are many different processes and diagrams in the EPOS model, it is likely that the dominant process responsible for such a two-hadron correlation pattern is the flux-tube fragmentation part of the EPOS model.

\section{EVENT-BY-EVENT EXCLUSIVE MEASUREMENTS TO STUDY FLUX-TUBE FRAGMENTATION DYNAMICS}

The comparison of the theoretical signatures with the experimental two-hadron correlation data in the last section reveals that there are $p_{T}$ regions in $p p$ collisions in which the flux-tube fragmentation process may dominate. We are therefore encouraged to examine the eventby-event dynamics of flux-tube fragmentation in these kinematic regions, in order to extract the wealth of information in the flux-tube fragmentation process [17. If the semi-classical description [5-10, 12] is a reasonable concept, then the local conservation laws and the rapidity-space-time ordering of hadrons provide a set of powerful tools to exhibit the chain of produced hadrons and to reconstruct the space-time coordinates of the $q-\bar{q}$ production vertices.

For an $e^{+} e^{-}$annihilation for which a single flux tube is produced, one can perform sphericity and thrust analysis to locate the longitudinal axis with respect to which the transverse masses and the rapidities of detected hadrons are determined. In a $p p$ collision, the conventional description of flux tube fragmentation is given in terms of two flux tubes, formed by the quark of one nucleon with the diquark of the other nucleon and vice versa [11. In another model of flux tubes in $p p$ collisions, the flux tubes arises from gluons in the color-glass condensate, and the number of flux tubes depend on the gluon transverse densities of the colliding hadrons [35,40].

For definiteness of our analysis in $p p$ collisions, we shall again follow the Lund semi-classical description in terms of the fragmentation of two colorless flux tubes so that deviations from such a semi-classical description will provide impetus for a search for alternative descriptions. The beam axis can be selected as the longitudinal axis with respect to which the transverse masses and rapidities are determined. The centers of momentum of the two flux tubes need not be the same. Thus, it will be necessary to order the two sets of hadrons according to their rapidities and decompose them as belonging to one set or the other of the fragmenting flux tubes. Each set of hadrons needs to be checked for energy, charge, and flavor conservation. The situation is not unlike the solution of a jig-saw puzzle where one makes use of the conservation of transverse momentum, flavor, and charge as hints to match the adjacent hadrons so as to link each flux tube by itself. By such a separation, one has two sets of hadrons each of which is likely to arise from the fragmentation of a single flux tube. We shall explore the tools that may facilitate the separation of two flux tubes in Section IX.

In the above procedure, care should be taken to allow for the possibility that the detected particles may be secondary hadrons arising from the feeding of heavier primary hadrons. We shall examine the fractions of higher meson resonances in Section VIII and Appendix A.

One works in the center-of-momentum system of the produced hadrons and arranges the primarily produced hadrons in the order of their rapidities: $y_{-m}<y_{-m+1}<$ $\ldots<y_{-2}<y_{-1}<\tilde{y}_{0}<y_{1}<y_{2}<. .<y_{n-1}<y_{n}$, where $\tilde{y}_{0}$ is closest to $y=0$.

The space-time coordinates $\left(u_{i}, v_{i}\right)$ of the $q-\bar{q}$ production vertices of the $q \bar{q}$ pairs for the production of the hadrons in a single flux tube can be obtained as follows:

1. To each hadron with transverse mass $m_{T i}$ and rapidity $y_{i}$, determine $\Delta v_{i}$ and $\Delta u_{i}$ by

$$
-\frac{\Delta v_{i}}{\Delta u_{i}}=-\frac{v_{i+1}-v_{i}}{u_{i+1}-u_{i}}=e^{-2 y_{i}}
$$$$
\text { and } \Delta u_{i}\left(-\Delta v_{i}\right)=\frac{m_{T i}^{2}}{\kappa^{2}} \text {. }
$$

The above two equations have the solution

$$
\left(\Delta u_{i}, \Delta v_{i}\right)= \pm \frac{m_{T i}}{\kappa}\left(e^{y_{i}},-e^{-y_{i}}\right)
$$

where the upper sign on the right-hand side is for positive $y_{i}$ and the lower sign for negative $y_{i}$.

2. We need to anchor one of the vertices, say the hadron with rapidity $\tilde{y}_{0} \sim 0$, to a space-time coordinate $\left(u_{0}, v_{0}\right)$ as determined from the measured $d N_{p p} / d y$. In the case of a Gaussian distribution, they are given by Eqs. (30) and (31) as a function of $y$ which is now set to $y=\tilde{y}_{0}$, with parameters $y_{0}, \sigma$, and $m_{T}$ as determined from other prior measurements of single-particle rapidity distributions.

3. Starting with the vertex at $\left(u_{0}, v_{0}\right)$ as shown in Fig. 4. we obtain the locations of other pair-production vertices in both directions of the longitudinal $z$-axis by the recursion formula

$$
\left(u_{i}, v_{i}\right)=\left(u_{i-1}, v_{i-1}\right)+\left(\Delta u_{i-1}, \Delta v_{i-1}\right)
$$

In the above reconstruction process, it should be kept in mind that as flux-tube fragmentation is only one of the many production mechanisms, not all collision events arise from flux-tube fragmentation. It may be necessary to consider each event case by case to see if it can be subject to the flux-tube fragmentation analysis. 


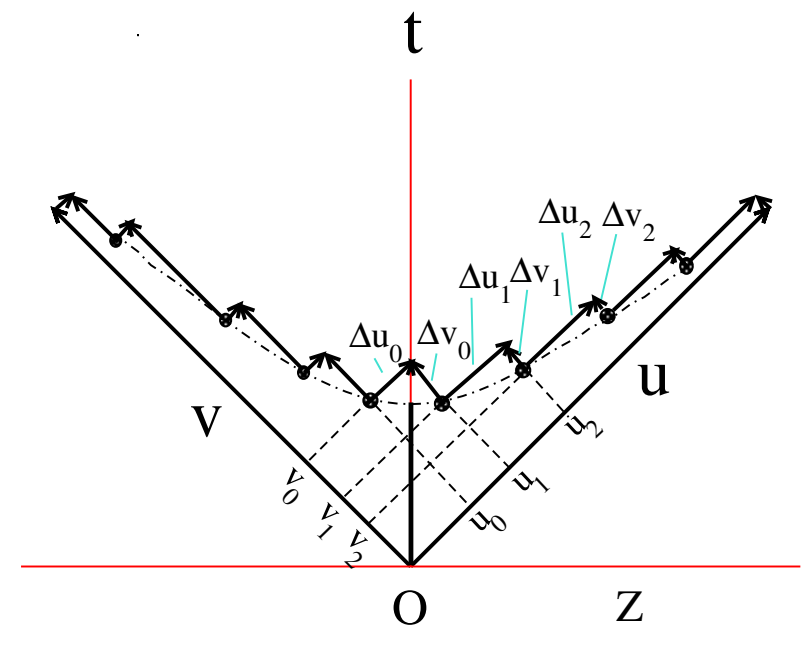

FIG. 4. (Color online). Shown here are the forward lightcone coordinate $u=z+t$ and backward light-cone coordinate $v=t-z$ for the reconstruction of space-time coordinates of vertices $\left(u_{i} v_{i}\right)$, where a $q$ and a $\bar{q}$ (represented by arrows) are produced. One starts with the vertex located at $\left(u_{0}, v_{0}\right)$ near $\tilde{y}_{0} \sim 0$ and use the recursion relation, $\left(u_{i}, v_{i}\right)=\left(u_{i-1}, v_{i-1}\right)+\left(\Delta u_{i-1}, \Delta v_{i-1}\right)$, to determine the other pair-production vertices.

\section{EXPERIMENTAL CHALLENGES}

There will be experimental challenges in the execution of the procedures outlined above. In a collision event with an appropriate trigger, there will be produced hadrons missing from detection, and the optimization of the detector will favor the detection of some hadrons more than some others. Neutrons, decay photons, and weak interaction products may also not be recorded. Nevertheless, with detection efficiency greater than $90 \%$ for $\pi^{-}$[54] (and perhaps slightly lower for other hadrons), there may remain an appreciable number of exclusive events sufficient for event-by-event analysis. One can alternatively lower the goal by examining the fragmentation only in a limited partial section of the flux tube (e.g. in the forward, backward, or central rapidity region). The examination of the many-body dynamics of even a partial section of the flux tube, no matter how short, remains useful in providing valuable information on the dynamics of the full flux-tube fragmentation process.

For those exclusive measurements with nearly no missing hadrons, there remain other complications. It is general thought that a large fraction of pions come from the decay of heavier mesons that are primarily produced in the hadronization process. The event-by-event analysis should be carried out with this possibility in mind.

The production of heavier mesons can be investigated by evaluating the invariant masses of combinations of detected lighter observed hadrons. The complexity of such a procedure depends on the number of produced hadrons and the fraction of heavier mesons, in comparison to the lighter mesons. It is therefore useful to have an idea on the fraction of heavier mesons that may be present by examining flux-tube fragmentation events with a standard Monte Carlo event generator.

For such an investigation, we use the the Lund fragmentation model in the event generator PYTHIA 6.4 [11, for hadron production at the sample energy of $\sqrt{s}=17.3 \mathrm{GeV}$ in the NA61/SHINE energy range, corresponding to a fixed target $p p$ collision at $p_{\text {lab }}=158$ $\mathrm{GeV} / \mathrm{c}$. In the default PYTHIA 6.4 program, the vector meson fraction involving $u$ and $d$ quarks is $(V /(V+P))_{u d} \equiv f_{V}^{u d}=\operatorname{PARJ}(11)=0.5$, the vector meson fraction involving the $s$ quark is $(V /(V+P))_{s} \equiv f_{V}^{s}=$ $\operatorname{PARJ}(12)=0.6$, and the strangeness suppression factor is $(s / u) \equiv f_{s}=\operatorname{PARJ}(2)=0.3$. One finds that a very large fraction of produced primary hadrons are $\rho, \omega, K$ and $K^{*}$ mesons.

Upon a careful examination and comparing with experimental measurement of heavier meson production, one finds that the primary vector and strange meson fractions may be over-estimated in the NA61/SHINE and SPS energy ranges in PYTHIA 6.4 with the default parameters, and a fine tuning appropriate for the range of energies of our interest may be needed. Previously in the production of $\rho^{0}, \omega$ and $K^{*}$ by $e^{+} e^{-}$annihilation in the $\Upsilon$ energy region at $\sqrt{s}\left(e^{+} e^{-}\right)=9.46$ to $10.02 \mathrm{GeV}$, a similar observation of an over-estimation with the default parameters was reached [65, using the Lund 7.3 Monte Carlo program that was adopted as part of the Lund fragmentation scheme in PYTHIA 6.4.

What then is the primary heavy meson fraction for the case of $p p$ collisions at $\sqrt{s} \sim 10$ to $20 \mathrm{GeV}$ at which flux tube fragmentation is expected to dominate? At $\sqrt{s}=17.3 \mathrm{GeV}$, the experimental observed heavier meson ratios are $\sigma_{\text {obs }}\left(\rho^{0}\right) / \sigma_{\text {obs }}\left(\pi^{-}\right)=0.12 \quad$ [62, 63,,$\sigma_{\text {obs }}\left(K^{-}\right) / \sigma_{\text {obs }}\left(\pi^{-}\right)$ $=0.08$ [66], and $\sigma_{\mathrm{obs}}\left(K^{*+}\right) / \sigma_{\mathrm{obs}}\left(K^{+}\right)=0.32$ [63]. As discussed in Appendix A, these observed ratios give the slightly modified primary ratios to be $\sigma_{\text {pri }}\left(\rho^{0}\right) / \sigma_{\text {pri }}\left(\pi^{-}\right)=0.19, \quad \sigma_{\text {pri }}\left(K^{-}\right) / \sigma_{\text {pri }}\left(\pi^{-}\right)=0.09$, and $\sigma_{\text {pri }}\left(K^{*+}\right) / \sigma_{\text {pri }}\left(K^{+}\right)=0.47$, after taking into account the feeding of heavier mesons. Using these primary ratios for the energy range of our interest, we find in Appendix A the heavy meson fractions $f_{V}^{u d}=0.20, f_{s}=0.14$, and $f_{V}^{s}=0.32$. These coefficients leads to dominant primary heavier meson fraction $f_{V}^{u d}$ only at the $20 \%$ level. With a multiplicity of order 10-15 in each event, the identification of the primary higher mass mesons at the NA61/SHINE and NICA energies may perhaps be a manageable task.

\section{SEPARATION OF THE TWO FLUX TUBES IN A $p p$ COLLISION}

At NA61/SHINE collision energies, Ref. [54 gives the rapidity distribution $d N\left(\pi^{-}\right) / d y$ in Fig. 2(b) as the sum of two Gaussian distributions with rapidity displacements at $y_{0}$ and $-y_{0}$. The two displaced Gaussian rapid- 
ity distributions can be considered as an indirect evidence for the presence of two flux tubes in $p p$ collisions. An event-by-event analysis may provide a way to discriminate the the possibilities of one, two, or many flux tubes.

We enlist below useful tools that may be utilized in the reconstruction of the flux tubes:

(1) Because of the conservation of baryon numbers, there will be two baryons in each $p p$ flux-tube fragmentation event. As leading particles, these baryon products can be used to anchor the two ends of the two flux tubes at opposite rapidities. Because neutrons are difficult to detect, one can choose events with two charged baryons to be the anchors at the opposite ends of the two flux tubes, and look for hadrons linking two separate tubes.

(2) Because of charge conservation in $q-\bar{q}$ pair production, a hadron adjacent to a charged hadron from the same tube is unlikely to have a charge of the same sign.

(3) To every produced strange hadron, there is an adjacent hadron of opposite strangeness from the same tube, on account of flavor conservation in $q-\bar{q}$ pair production. The flavor quantum numbers of the chain of hadrons from a tube are aligned. The flavor of the quark in a hadron and the flavor of the antiquark of the hadron adjacent in rapidity are the same in the semi-classical picture. Such a correlation may be modified by quantum and other fluctuation mechanism, and experimental measurements of such correlations are sorely needed for a quantitative assessment.

(4) In the flux-tube fragmentation under the pair production mechanism, a hadrons adjacent to each other in the flux tube is likely to be correlated azimuthally at large opening angles close to $\pi$ because the produced $q$ and $\bar{q}$ are produced back-to-back azimuthally in the pair frame. As the transverse momenta of the other spectator quark and antiquark are not necessarily zero, the back-to-back azimuthal correlation is reduced to be a correlation at a large opening azimuthal angle close to $\pi$.

(5) If the rapidity centers of hadrons produced by two flux tubes are displaced by $-y_{0}$ and $+y_{0}$, as given in Eq. (34) by [54, then the two flux tubes will produced hadrons that are displaced from each other in rapidity. The rapidity displacement $y_{0}$ increases with the collision energy, as indicated in Table II. As a consequence, the sections of hadrons of the two flux tubes that overlap in rapidity is smaller, the greater the displacement of the two flux tube rapidities $y_{0}$ and the easier it is to separate the two flux tubes. On the other hand, the greater the collision energy, the greater the multiplicity and the more difficult it is to reconstruct the dynamics with a greater number of possible combinations.

With this set of tools, it may be possible to resolve some of the puzzles of the hadron configuration for some of the $p p$ collision events. Because no such data have been developed as yet, the knowledge of some explicit spacetime dynamics in some events serves well in extending our understanding of the hadronization dynamics of the particle production process.

\section{INSIDE-OUTSIDE CASCADE OR OUTSIDE-INSIDE CASCADE?}

An explicit reconstruction of the many-body correlations provides a valuable tool to distinguish the fine details of different reaction mechanisms of flux-tube fragmentation in high-energy $e^{+} e^{-}$annihilations and $p p$ collisions.

There is the outside-inside cascade picture of particle production in flux-tube fragmentation that comes from perturbative QCD in which the outgoing quark and antiquark (or diquark) are represented by longitudinal jets of partons, and hadrons are produced by parton cascading from the jets. Low energy hadrons arises from the "wee" partons which could form a bridge and neutralize the quark color charges with those from the cascading of the other jet. This is the outside-inside cascade picture of the longitudinal production of soft particles.

An alternative view that has been suggested by Bjorken, Casher, Kogut, and Suskind [2, 3] is the insideoutside longitudinal cascade in which the receding quark and antiquark pair interact by the exchange of a vector interaction (gluon exchange), and these interactions lead to the production of particles from the inside out.

The explicit display of the dynamics as a result of the flux-tube fragmentation will allow an assessment of the mechanism of particle production. The difference between the inside-outside and outside-inside picture shows up as a difference in the correlation of the soft particles, those with small rapidities. In the inside-outside cascade picture, the hadrons in the small rapidity region are correlated because they share the process of nonperturbative particle production, while in the outside-inside cascade, these particles are not correlated as they come from independent cascade of the leading partons. At the time when the soft particles are produced, the leading partons are so far apart that there is little chance for these wee partons to overlap in coordinate space to correlate.

The two-particle angular correlation with opposite charges may appear to lend support to the picture of inside-outside cascade for flux-tube fragmentation for low- $p_{T}$ particle production [48. The situation may depend on the transverse momentum $p_{T}$ of the produced particles, as the hard process with high- $p_{T}$ particles may favor the outside-inside cascade picture of particle production. A many-body correlation will be able to reveal whether the picture of inside-outside or outside-inside cascade will depend on the $p_{T}$ domain of the detected particles.

\section{CONCLUSION AND DISCUSSIONS}

There are many mechanisms of particle production in $p p$ collisions, such as flux-tube fragmentation [1-21, hard-scattering [22 28], direct fragmentation [34], colorglass condensate [35, 36], EPOS model [41], and Landau hydrodynamics [2 47]. Each of the proposed theoretical 
production mechanisms exhibits specific space-time dynamics on an event-by-event basis. If a substantial fraction of the produced particles are detected in the collision event, then the event-by-event dynamics of the produced particles may be utilized to identify the particle production mechanism that has occurred in that event, not only to test and discriminate theoretical descriptions but also to provide important pieces of information on the production process. It is therefore useful to develop methods to analyze event-by-event dynamics of produced hadrons in order to extract the wealth of information they provide. For clarity, focus of physical principles, and a better theoretical understanding, we have examined the flux-tube fragmentation process with analytical models and simplifying assumptions against which refinement and modifications may be brought forth for a comprehensive description of the production and hadronization process.

Successes in carry out the event-by-event analysis in flux-tube fragmentation may stimulate similar exploration to investigate the space-time dynamics in other reaction mechanisms such as the space-time dynamics in a hard-scattering process in which hadrons from azimuthally back-to-back jets are expected and the dynamics of the showering process are displayed. Similarly, a hydrodynamical occurrence in a collision event with a large number of produced particles will exhibit specific patterns in an event-by-event basis [44, 45]. The simple event-by-event analysis in flux-tube fragmentation in $p p$ may assist the investigation of the partition of the two flux tubes collisions, the interference and interaction between flux tubes, the possible merging of the flux tubes, and the description of the multiple collision processes in $p A$ and $A A$ collisions.

In $p p$ collisions at $\sqrt{s}=6-200 \mathrm{GeV}$, the gross features of the experimental NA61/SHINE [55] 58] and STAR [4952 angular correlation data for two low- $p_{T}$ hadrons with opposite charges match the signature of the fragmentation of a flux tube 48. Thus $p p$ collisions in the energy range of the NICA facility and NA61/SHINE energy scan are favorable for the study of the many-body correlation for flux-tube fragmentation discussed here.

Exclusive measurements has the difficulties that there will be particles missing from detection, and the optimization of the detector will favor the detection of some hadrons more than some others. It is an experimental question whether there remains an appreciable fractions of events in which all (or almost all) produced hadrons may be recorded. If this is difficult to achieve, a lower goal of looking only for the fragmentation process in a partial section of the whole flux tube in the forward, backward, or central rapidity region, may be carried out with the procedures outlined in Section VII. The ability to examine the many-body dynamics of even a partial section of the flux tube may be a useful asset. It will pave the way for a full investigation of the full flux tube when detection conditions may become more favorable.

With regard to the rapidity-space-time ordering that is expected semi-classically, there has not been much study on the quantum fluctuation of such an ordering. The explicit event-by-event measurement will provide useful information on how the flux-tube fragmentation will be affected by stochastic and quantum fluctuations.

If confirmed experimentally in $p p$ collisions, the rapidity-space-time ordering may be used as a strong signature of flux-tube fragmentation that may be applied to the search for the possible presence of the remnants of cosmic flux tubes in the early history of the universe. One can envisage that at the end-point of the expansion phase of the cosmic string dynamics, the cosmic strings will fragment into cosmic mass entities. If the analogy of color string fragmentation is a good guide, the longitudinal velocities or rapidities of cosmic mass entities will be ordered longitudinally along the string axis and adjacent cosmic masses may be correlated azimuthally with back-to-back transverse momenta. A measurement of the angular correlation between cosmic masses and the momenta of a longitudinal chain of masses may provide a glimpse of the space-time dynamics of the prior flux tube configuration and a test of the concept of knotty/linked flux tubes proposed recently [59].

\section{Acknowledgements}

The author would like to thank Drs. David Blaschke, Elena Kokoulina, and Ken Read for helpful discussions and to Prof. A. Berera for bringing attention to the knotty/linked flux tubes in cosmological inflation. The research was supported in part by the Division of Nuclear Physics, U.S. Department of Energy under Contract DEAC05-00OR22725.

\section{Appendix A: Vector meson fractions and the Strangeness suppression factor}

Experimental measurements in $p p$ collisions and $e^{+}-e^{-}$annihilations give ratios of observed yields $\sigma_{\text {obs }}\left(\rho^{0}\right) / \sigma_{\text {obs }}\left(\pi^{-}\right), \quad \sigma_{\text {obs }}\left(K^{-}\right) / \sigma_{\text {obs }}\left(\pi^{-}\right), \quad$ and $\sigma_{\text {obs }}\left(K^{*-}\right) / \sigma_{\text {obs }}\left(K^{-}\right)$where the subscript "obs" stands for observed hadron quantities, to distinguished from the primary hadron quantities with the subscript "pri". For $p p$ collisions involving flux-tube fragmentation, the ratios of neutral or negative hadrons are less likely to arise from leading projectile and target fragmentations and are more likely to come from flux-tube fragmentation. We would like to examine those yield ratios involving neutral and negative hadrons within the mechanism of flux-tube fragmentation.

To infer the primary yield ratios within the flux-tube fragmentation mechanism, we need to take into account the feeding from the heavier mesons in $\rho \rightarrow \pi \pi, \omega \rightarrow \pi \pi \pi$ and $K^{*} \rightarrow(K \pi)$. The observed and the primary cross 
sections are related by

$$
\begin{aligned}
\sigma_{\mathrm{obs}}\left(\pi^{+}+\pi^{0}+\right. & \left.\pi^{-}\right)=\sigma_{\mathrm{pri}}\left(\pi^{+}+\pi^{0}+\pi^{-}\right) \\
& +2 \sigma_{\mathrm{pri}}\left(\rho^{+}+\rho^{-}+\rho^{0}\right)+3 \sigma_{\mathrm{pri}}(\omega) \\
& +\sigma_{\mathrm{pri}}\left(K^{*+}+K^{*-}+K^{* 0}+\bar{K}^{* 0}\right) \\
\sigma_{\mathrm{obs}}\left(K^{+}+K^{-}+\right. & \left.K^{0}+\bar{K}^{0}\right)=\sigma_{\mathrm{pri}}\left(K^{+}+K^{-}+K^{0}+\bar{K}^{0}\right) \\
& +\sigma_{\mathrm{pri}}\left(K^{*+}+K^{*-}+K^{* 0}+\bar{K}^{* 0}\right) .
\end{aligned}
$$

The ratios of the primary yields can then be used to determine the vector meson fractions $f_{V}^{u d}$ and $f_{V}^{s}$, and the strangeness suppression factor $f_{s}$. They are defined as

$$
\begin{aligned}
f_{V}^{u d} & =\left(\frac{V}{V+P}\right)_{u d} \\
& =\frac{\sigma_{\mathrm{pri}}\left(\rho^{+}+\rho^{-}+\rho^{0}+\omega\right)}{\sigma_{\mathrm{pri}}\left(\pi^{+}+\pi^{0}+\pi^{-}+\rho^{+}+\rho^{-}+\rho^{0}+\omega\right)}, \\
f_{V}^{s} & =\left(\frac{V}{V+P}\right)_{s} \\
& =\frac{\sigma_{\mathrm{pri}}\left(K^{*+}+K^{*-}+K^{* 0}+\bar{K}^{* 0}\right)}{\sigma_{\mathrm{pri}}\left(K^{+}+K^{-}+K^{0}+\bar{K}^{0}+K^{*+}+K^{*-}+K^{* 0}+\bar{K}^{* 0}\right)}, \\
f_{s} & =\frac{\sigma_{\mathrm{pri}}\left(K^{+}+K^{-}+K^{0}+\bar{K}^{0}+K^{*+}+K^{*-}+K^{* 0}+\bar{K}^{* 0}\right)}{\sigma_{\mathrm{pri}}\left(\pi^{+}+\pi^{0}+\pi^{-}+\rho^{+}+\rho^{-}+\rho^{0}+\omega\right)} .
\end{aligned}
$$

We assume that in a $p p$ collision, the fragmentation of the leading charged hadrons have been taking into account, and we are left to deal with a neutral quark-antiquark color flux tube (or tubes), for which $\quad \sigma_{\text {pri }}\left(\rho^{+}\right)=\sigma_{\text {pri }}\left(\rho^{0}\right)=\sigma_{\text {pri }}\left(\rho^{-}\right)=\sigma_{\text {pri }}(\omega)$, $\sigma_{\text {pri }}\left(K^{ \pm}\right)=\sigma_{\text {pri }}\left(K^{0}\right)=\sigma_{\text {pri }}\left(\bar{K}^{0}\right), \quad$ and $\quad \sigma_{\text {pri }}\left(K^{* \pm}\right)=$ $\sigma_{\text {pri }}\left(K^{* 0}\right)=\sigma_{\text {pri }}\left(\bar{K}^{* 0}\right)$. After taking into account the feeding from the heavier mesons to of lighter mesons, we obtain for the fragmentation of a neutral color flux tube

$$
\begin{gathered}
\frac{\sigma_{\mathrm{pri}}\left(\rho^{0}\right)}{\sigma_{\mathrm{pri}}\left(\pi^{-}\right)}=\frac{\sigma_{\mathrm{obs}}\left(\rho^{0}\right) / \sigma_{\mathrm{obs}}\left(\pi^{-}\right)}{1-3 \sigma_{\mathrm{obs}}\left(\rho^{0}\right) / \sigma_{\mathrm{obs}}\left(\pi^{-}\right)} \\
\times\left(1+\frac{f_{V}^{s}}{1-f_{V}^{s}} \frac{4}{3} \frac{\sigma_{\mathrm{pri}}\left(K^{-}\right)}{\sigma_{\mathrm{pri}}\left(\pi^{-}\right)}\right), \\
\frac{\sigma_{\mathrm{pri}}\left(K^{-}\right)}{\sigma_{\mathrm{pri}}\left(\pi^{-}\right)}=\frac{\left(1+\frac{9}{4} \frac{f_{V}^{u d}}{\left(1-f_{V}^{u d}\right)}\right) \frac{\sigma_{\mathrm{obs}}\left(K^{-}\right)}{\sigma_{\mathrm{obs}}\left(\pi^{-}\right)}}{\frac{1}{1-f_{V}^{s}}-\frac{4}{3} \frac{f_{V}^{s}}{1-f_{V}^{s}} \frac{\sigma_{\mathrm{obs}}\left(K^{-}\right)}{\sigma_{\mathrm{obs}}\left(\pi^{-}\right)}}, \\
\frac{\sigma_{\mathrm{pri}}\left(K^{*-}\right)}{\sigma_{\mathrm{pri}}\left(K^{-}\right)}=\frac{\frac{\sigma_{\mathrm{obs}}\left(K^{*-}\right)}{\sigma_{\mathrm{obs}}\left(K^{-}\right)}}{1-\frac{\sigma_{\mathrm{obs}}\left(K^{*-}\right)}{\sigma_{\mathrm{obs}}\left(K^{-}\right)}},
\end{gathered}
$$

where

$$
\begin{gathered}
f_{V}^{u d}=\left(\frac{V}{V+P}\right)_{u d}=\frac{\frac{4}{3} \frac{\sigma_{\mathrm{pri}}\left(\rho^{0}\right)}{\sigma_{\mathrm{pri}}\left(\pi^{-}\right)}}{1+\frac{4}{3} \frac{\sigma_{\mathrm{pri}}\left(\rho^{0}\right)}{\sigma_{\mathrm{pri}}\left(\pi^{-}\right)}}, \\
f_{V}^{s}=\frac{\frac{\sigma_{\mathrm{pri}}\left(K^{*-}\right)}{\sigma_{\mathrm{pri}}\left(K^{-}\right)}}{1+\frac{\sigma_{\mathrm{pri}}\left(K^{*-}\right)}{\sigma_{\mathrm{pri}}\left(K^{-}\right)}},
\end{gathered}
$$

$$
f_{s}=\frac{1-f_{V}^{u d}}{1-f_{V}^{s}} \frac{4}{3} \frac{\sigma_{\mathrm{pri}}\left(K^{-}\right)}{\sigma_{\mathrm{pri}}\left(\pi^{-}\right)} .
$$

This set of equations allow the determination of the primary vector meson fraction and strangeness suppression factor from the observed yield ratios in flux-tube fragmentation by iteration.

The $\rho^{0}$ and $\pi^{-}$meson yields in $p p$ collisions at NA61/SHINE and SPS energies have been measured experimentally 6063 . At $\sqrt{s}=6.84 \mathrm{GeV}$, the observed $\sigma_{\text {obs }}\left(\rho^{0}\right) / \sigma_{\text {obs }}\left(\pi^{-}\right)$has been found to be about 0.07 [60]. At $\sqrt{s} \sim 19.7 \mathrm{GeV}$, it rises to $0.12 \pm 0.3$, but corrections to the background $K-\pi$ correlations in $\pi-\pi$ correlation measurements reduce this observed $\rho^{0} / \pi^{-}$ratio to $0.09 \pm 0.03$ 62, 63. At higher energies up to $\sqrt{s} \sim 60 \mathrm{GeV}$, the ratio $\sigma_{\text {obs }}\left(\rho^{0}\right) / \sigma_{\text {obs }}\left(\pi^{-}\right)$becomes nearly flat at about 0.13 (See Fig. 5 of Ref. 61]). These ratios are consistent with the multiplicity ratio $\left\langle\rho^{0}\right\rangle /\left\langle\pi^{-}\right\rangle$in $e^{+}-e^{-}$annihilation, which gives $\left\langle\rho^{0}\right\rangle /\left\langle\pi^{-}\right\rangle=\{0.109,0.138,0.138\}$ at $\sqrt{s}\left(e^{+} e^{-}\right)=\{10,25-35,91\} \mathrm{GeV}$, respectively 64.

Experimental measurements in 63] give an observed $\sigma_{\text {obs }}\left(K^{*-}\right) / \sigma_{\text {obs }}\left(K^{0}\right)=0.32$ (error $\left.+0.14,-0.18\right)$ and a strange vector meson fraction $f_{V}^{s}=0.32$ for $p p$ collisions at $\sqrt{s}=19.7 \mathrm{GeV}$. Such a ratio is consistent with the multiplicity ratio in $e^{+}-e^{-}$annihilations, which give observed $\left\langle K^{*+}\right\rangle /\left\langle K^{+}\right\rangle=(0.29 \pm 0.04,0.43 \pm 0.04)$ at $\sqrt{s}\left(e^{+} e^{-}\right)=(10.45,29) \mathrm{GeV}$, respectively (Table 18 of 65]).

In $p p$ collisions at NA61/SHINE energies, the ratio $K^{+} / \pi^{+}$is greater than $K^{-} / \pi^{-}$. At these low energies the production of $K^{+}$and $\pi^{+}$is influenced by contributions from baryon resonance production in which an incident proton is excited to a baryon resonance that subsequently emits a meson [56, 57]. The baryon resonances are produced mostly at the projectile and target fragmentation regions but have contributions at central rapidity when the collision energies are low. On the other hand, negative mesons such as $K^{-}$and $\pi^{-}$are more likely to arise from flux-tube fragmentation rather than projectile or target fragmentation. The ratio $K^{-} / \pi^{-}$can be a better gauge of the strangeness suppression in flux-tube fragmentation.

At $\sqrt{s} \sim 3 \mathrm{GeV}$, the ratio $K^{-} / \pi^{-}$is zero because the threshold for $K^{-}$production is higher than the threshold for $\pi^{-}$production. At $\sqrt{s}=17.3 \mathrm{GeV}$, $K^{-} / \pi^{-}$rises to about 0.08 . At $\sqrt{s}=200 \mathrm{GeV}$, one finds $\sigma_{\text {obs }}\left(K^{+}\right) \sim \sigma_{\text {obs }}\left(K^{-}\right)$and $\sigma_{\text {obs }}\left(\pi^{+}\right) \sim \sigma_{\text {obs }}\left(\pi^{-}\right)$, with $\sigma_{\text {obs }}\left(K^{+}\right) / \sigma_{\text {obs }}\left(\pi^{+}\right) \sim \sigma_{\text {obs }}\left(K^{-}\right) / \sigma_{\text {obs }}\left(\pi^{-}\right) \sim 0.1$ [67]. At higher energies, the ratio $\sigma_{\mathrm{obs}}\left(K^{-}\right) / \sigma_{\mathrm{obs}}\left(\pi^{-}\right)$become relatively flat at 0.12 (see Fig. 7 of [66]).

As an example, we can consider the case of $\sqrt{s}=17.3$ $\mathrm{GeV}$ at which $\sigma_{\mathrm{obs}}\left(\rho^{0}\right) / \sigma_{\text {obs }}\left(\pi^{-}\right)=0.1262$, 63. $\quad \sigma_{\text {obs }}\left(K^{*+}\right) / \sigma_{\text {obs }}\left(K^{+}\right)=0.32 \quad$ 63], and $\sigma_{\text {obs }}\left(K^{-}\right) / \sigma_{\text {obs }}\left(\pi^{-}\right)=0.08$ 66]. We find from Eqs. (A1)-(A3) the ratios of primary yields to be $\sigma_{\text {pri }}\left(\rho^{0}\right) / \sigma_{\text {pri }}\left(\pi^{-}\right)=0.19, \quad \sigma_{\text {pri }}\left(K^{-}\right) / \sigma_{\text {pri }}\left(\pi^{-}\right)=0.09$ [66], and $\sigma_{\text {pri }}\left(K^{*+}\right) / \sigma_{\text {pri }}\left(K^{+}\right)=0.47$. From Eqs. (A4)-(A6), these primary ratios give $f_{V}^{u d}=0.20, f_{V}^{s}=0.32$, and $f_{s}=0.14$. 
[1] Y. Nambu, Lectures at Copenhagen Symposium (1970).

[2] J. D. Bjorken, Lectures presented at the 1973 Proceedings of the Summer Institute on Particle Physics, edited by Zipt, SLAC-167 (1973).

[3] A. Casher, J. Kogut, and L. Susskind, Phys. Rev. D 10, 732 (1974); A. Casher, H. Neuberger, and S. Nussinov, Phys. Rev. D 20179 (1979); Phys. Rev. D 211966 (1980).

[4] J. Schwinger, Phys. Rev. 128, 2425 (1962); J. Schwinger, in Theoretical Physics, Trieste Lectures, 1962 (I.A.E.A., Vienna, 1963), p. 89.

[5] X. Artru and G. Mennessier, Nucl. Phys. B70, 93 (1974).

[6] B. Andersson, G. Gustafson and C. Peterson, Z. Phys. C1, 105 (1979); B. Andersson, G. Gustafson and B. Söderberg, Z. Phys. C20, 317 (1983).

[7] A comprehensive review of the application of the Flux Tube Fragmentation Model for nucleon-nucleon and $e^{+}-$ $e^{-}$collisions can be found in B. Andersson, G. Gustafson, G. Ingelman, and T. Sjöstrand, Phys. Rep. 97, 31 (1983), and X. Artru, Phys. Rep. 97, 147 (1983).

[8] X. Artru, Z. Phys. C26, 83 (1984).

[9] B. Andersson, G. Gustafson, and T. Sjöstrand, Zeit. für Phys. C20, 317 (1983); T. Sjöstrand and M. Bengtsson, Computer Physics Comm. 43, 367 (1987); B. Andersson, G. Gustavson, and B. Nilsson-Alqvist, Nucl. Phys. B281, 289 (1987).

[10] T. Sjöstrand, Comp. Phys. Comm. 39, 347 (1986); T. Sjöstrand, and M. Bengtsson, Comp. Phys. Comm. 43, 367 (1987).

[11] T. Sjöstrand et at., PYTHIA 6.4 Physics and Manual, JHEP 05, 026 (2006), arXiv:hep-ph/0603175.

[12] T. Sjöstrand et al., An Introduction to PYTHIA 8.2, Comp. Phys. Comm. 191, 159 (2015), arXiv:1410.3012

[13] J. Schwinger, Phys. Rev. 82, 664 (1951).

[14] R. C. Wang and C. Y. Wong, Phys. Rev. D38, 2890 (1988).

[15] H-P. Pavel and D. Brink, Zeit. Phys. C51, 119 (1991).

[16] C. Y. Wong, R. C. Wang, and C. C. Shih, Phys. Rev. D44, 257 (1991).

[17] C. Y. Wong, R. C. Wang, Phys. Rev. D44, 679 (1991).

[18] G. Gatoff and C.Y. Wong, Phys. Rev. D 46, 997 (1992); and C. Y. Wong and G. Gatoff, Phys. Rep. 242, 1994, 489 (1994).

[19] C. Y. Wong, R. C Wang, and J. S. Wu, Phys. Rev D51, 3940 (1995).

[20] D.A. Derkach, G.A. Feofilov, Phys. Atom. Nucl. 71, 2087 (2008); E.O. Bodnya, V.N. Kovalenko, A.M. Puchkov, G.A. Feofilov, AIP Conf.Proc. 1606, 273 (2014); J. Adam et al. JHEP 1505 (2015); G. Feofilov, I. Altsybeev, O. Kochebina, XXII International Baldin Seminar on High Energy Physics Problems 15-20 September, 2014 JINR, Dubna, Russia, PoS(Baldin ISHEPP XXII) 067.

[21] C. Y. Wong, Introduction to High-Energy Heavy-Ion Collisions, World Scientific Publisher, 1994.

[22] R. Blankenbecler and S. J. Brodsky, Phys. Rev. D 10, 2973 (1974); R. Blankenbecler, S. J. Brodsky and J. Gunion, Phys. Rev. D 12, 3469 (1975); E. A. Schmidt and R. Blankenbecler, Phys. Rev. D 15, 3321 (1977).

[23] A.L.S. Angelis et al. (CCOR Collaboration), Phys. Lett. B 79, 505 (1978).
[24] R. P. Feynman, R. D. Field and G. C. Fox, Phys. Rev. D 18, 3320 (1978).

[25] J. F. Owens, E. Reya, and M. Glück, Phys. Rev. D 18, 1501 (1978); D. W. Duke, J. F. Owens, Phy. Rev. D 30, 49 (1984).

[26] J. Rak and M. J. Tannenbaum, High- $p_{T}$ Physics in the Heavy Ion , Cambridge University Press, Cambridge, 2013.

[27] Ellis, R K; Stirling, W J and Webber, B R (1996). QCD and Collider Physics. Camb. Monogr. Part. Phys. Nucl. Phys. Cosmol., Volume 8. Cambridge University Press, Cambridge. ISBN 0521581893.

[28] C.Y. Wong, G. Wilk, Acta Physica Polonica B42, 2047 (2012). C.Y. Wong, G. Wilk, Phys. Rev. D 87, 114007 (2013). C.Y. Wong, G. Wilk, L.J.L. Cirto, C.Tsallis, EPJ Web Conf.90, 04002 (2015) . C.Y. Wong, G. Wilk, L.J.L. Cirto, C.Tsallis, Phys. Rev. D91 114027 (2015).

[29] C. Y. Wong, R. C. Wang, and C. C. Shih, Phys. Rev. D44, 257 (1991).

[30] E. Abdalla, M. C. B. Abdalla, and K. D. Rothe, Two Dimensional Quantum Field Theory, World Scientific Publishing Company, Singapore, 2001.

[31] Y. Frishman and J. Sonnenschein, Phys. Rep. 223, 309 (1993); Y. Frishmann, A. Hanany, and J. Sonnenschein, Nucl. Phy. B429, 75 (1994); A. Armonic and J. Sonnenschein, Nucl. Phy. B457, 81 (1995); A. Armonic, Y. Frishmann, J. Sonnenschein, and U. Trittmann Nucl. Phy. B537, 503 (1999); A. Abrashikin, Y. Frishmann, and J. Sonnenschein, Nucl. Phy. B703, 320 (2004).

[32] C. Y. Wong, Phys. Rev. C81, 064903 (2010).

[33] A. V. Koshelkin and C. Y. Wong, Phy. Rev D 86, 125026 (2012).

[34] C. Y. Wong and R. Blankenbecler, Phys. Rev. C22, 2433 (1980).

[35] L. McLerran and R. Venugopalan, Phys. Rev. D50, 2225 (1994); J. Jalilian-Marian, A. Kovner, L. McLerran and H. Weigert, Phys. Rev. D55, 5414 (1997).

[36] L. McLerran, "The Color Glass Condensate and Small x Physics: 4 Lectures", Lect.Notes Phys. 583, 291, 2002, DOI:10.1007/3-540-45792-5_8 (arxiv:hep-ph/0104285).

[37] L. McLerran, "The CGC and the Glasma: Two Lectures at the Yukawa Insitute", Prog. Theor. Phys. Suppl. 187, 17 (2011), arXiv:1011.3204.

[38] L. McLerran, "Strongly Interacting Matter Matter at Very High Energy Density: 3 Lectures in Zakopane", Acta Phys. Polon. B41, 2799 (2010), arXiv:1011.3203.

[39] A. Kovner, L. McLerran, and H. Weigert, Phys. Rev. D52, 3809 (1995); A. Kovner, L. McLerran, and H. Weigert, Phys. Rev. D52, 6231 (1995).

[40] F. Geilis, T. Lappi, and L. McLerran, Nucl. Phys. A828, 149 (2009); K. Fukushima, F. Gelis, and T. Lappi, Nucl. Phys. A831, 184 (2009); F. Gelis and N. Tanji, Prog. in Part. Nucl. Phys. 87, 1 (2016) (DOI:10.1016/j.ppnp.2015.110001, arXiv:1510.05451 (2015)).

[41] K. Werner, F. M. Liu and T. Pierog, Phys. Rev. C 74, 044902 (2006). hep-ph/0506232.

[42] L. D. Landau, Izod. Akad. Nauk SSSR 17, 51 (1953); S. Z. Belenkij and L. D. Landau, Usp. Fiz. Nauk 56, 309 (1955); I. M. Khalatnikov, Zh. Eksp. Teor. Fiz. 27, 529 (1954). 
[43] C. Y. Wong, Phys. Rev. C78, 054902 (2008);

[44] A. Sen, J. Gerhard, G. Torrieri, K. Read, C. Y. Wong, Phys. Rev. C 91, 024901 (2015)

[45] C. Y. Wong, A. Sen, J. Gerhard, G. Torrieri, K. Read Phys. Rev. C 90, 064907 (2014).

[46] M. Murray for the Brahms Collaboration, J. Phys. G. 30, S667 (2004); I. G. Bearden et al. (BRAHMS Collaboration), Phys. Rev. Lett. 94, 162301 (2005); M. Murray for the Brahms Collaboration, J. Phys. G 35, 044015 (2008).

[47] P. Steinberg, Nucl. Phys. A 752, 423 (2005).

[48] C. Y. Wong, Phys. Rev. D 92, 074007 (2015).

[49] J. Adams et al. (STAR Collaboration), Phys. Rev. C 74, 032006 (2006).

[50] R. J. Porter and T. A. Trainor, (STAR Collaboration), J. Phys. Conf. Ser. 27, 98 (2005).

[51] T. A. Trainor and R. L. Ray, Phys. Rev. C 84, 034906 (2011).

[52] R. L. Ray, Phys. Rev. D 84, 034020 (2011); T. A. Trainor and D. J. Prindle, Improved isolation of the pp underlying event based on minimum-bias trigger-associated hadron correlations, arXiv:1310.0408 [hep-ph].

[53] T. A. Trainor and D. T. Kettler, Phys. Rev. C84, 024910 (2011), arXiv:1010.3048

[54] N. Abgrall et al. (NA61/SHINE Collaboration Eur. Phys. J. C74, 2794 (2014) (arXiv:1310.2417).
[55] M. Maksiak (NA61/SHINE Collaboration), arXiv: 1503.02470

[56] M. Gazdzicki (NA61/SHINE Collaboration), EPJ Web Conf. 95, 01005 (2015), arXiv:1412.4243.

[57] D. T. Larsen (NA61/SHINE Collaboration), Proceedings of International Conference on Strange Quark Matter, Dubna, July 6-11, 2015 (to be published).

[58] A. Seryakov (NA61/SHINE Collaboration), Proceedings of International Conference on Strange Quark Matter, Dubna, July 6-11, 2015 (to be published).

[59] A. Berera, R. V. Buniy, T. W. Kephart, H. Päs, J. G. Rosa, arXiv:1508.01458 (2015).

[60] V. Blobel et al., Phys. Lett. B48, 73 (1974).

[61] M.G.Albrow et al., Nucl. Phys. B155, 39 (1979).

[62] R. Singer et al., Phys. Lett. 60B, 385 (1976).

[63] R. Singer et al., Nucl. Phys. B135, 265 (1978).

[64] K. A. Olive et al., (Particle Date Group), Chin. Phy. C38, 1 (2014), Table 50.1, p. 532, \{ Average HadronMultiplicities in Hadronic e+e Annihilation Events\}.

[65] H. Albrecht et al. (ARGUS Collaboration), Zeit. fur Phys. C 61, 1 (1994).

[66] S. Pulawski (NA61/SHINE Collaboration), PoS CPOD2014 010 (2015), arXiv:1502.07916

[67] J. Adams et al. (STAR Collaboration) Phys. Rev. Lett. 92, $112301(2004)$ 